\title{
Spectral dependence of purely-Kerr-driven filamentation in air and argon
}

\author{
W. Ettoumi, ${ }^{1}$ P. Béjot ${ }^{2}$ Y. Petit, ${ }^{1}$ V. Loriot,,${ }^{2,3}$ E. Hertz, ${ }^{2}$ O. Faucher, ${ }^{2}$ B. Lavorel, ${ }^{2}$ J. Kasparian,,${ }^{1,}$ and J.-P. Wolf ${ }^{1}$ \\ ${ }^{1}$ Université de Genève, GAP-Biophotonics, 20 rue de l'Ecole de Médecine, 1211 Geneva 4, Switzerland \\ ${ }^{2}$ Laboratoire Interdisciplinaire Carnot de Bourgogne (ICB), UMR 5209 CNRS-Université de Bourgogne, 9 Av. A. Savary, \\ Boîte Postale 47 870, F-21078 DIJON Cedex, France \\ ${ }^{3}$ Instituto de Química Física Rocasolano, CSIC, C/Serrano, 119, 28006 Madrid, Spain
}

(Received 4 May 2010; published 21 September 2010; publisher error corrected 27 September 2010)

\begin{abstract}
Based on numerical simulations, we show that higher-order nonlinear indices (up to $n_{8}$ and $n_{10}$, respectively) of air and argon have a dominant contribution to both focusing and defocusing in the self-guiding of ultrashort laser pulses over most of the spectrum. Plasma generation and filamentation are therefore decoupled. As a consequence, ultraviolet wavelength may not be the optimal wavelength for applications requiring to maximize ionization.
\end{abstract}

DOI: 10.1103/PhysRevA.82.033826

PACS number(s): 42.65.Jx, 42.65.Tg, 78.20.Ci

The filamentation of high-power ultrashort laser pulses is generally described as a dynamic balance between Kerr selffocusing and the defocusing by the plasma generated at the nonlinear focus [1-5]. In this description, the Kerr-induced change in the refractive index is truncated to the first term $n_{2} I$, where $I$ is the local intensity. However, at higher intensities, the development has to be extended to higher-order terms in $I$ so that the real part of the refractive index at any frequency $\omega$ writes

$$
n(\omega)=n_{0}(\omega)+\Delta n_{\mathrm{Kerr}}(\omega)=n_{0}(\omega)+\sum_{j \geqslant 1} n_{2 j}(\omega) I^{j},
$$

where the $n_{2 j}(\omega)$ coefficients are related to the $(2 j+1)$ thorder susceptibility tensor $\chi^{(2 j+1)}(\omega)$, in the degenerate case where all considered fields are at frequency $\omega$ :

$$
n_{2 j}(\omega)=\frac{(2 j+1) !}{2^{j+1} j !(j+1) !} \frac{1}{\left[n_{0}^{2}(\omega) \epsilon_{0} c\right]^{j}} \operatorname{Re}\left[\chi^{(2 j+1)}(\omega)\right] .
$$

In the past years, several numerical works have investigated the influence of the quintic nonlinear response on the filamentation dynamics at a wavelength of $800 \mathrm{~nm}$, although without knowledge of its actual value [6-10]. They suggested that this term was defocusing, but considered it as marginal. Recently, the measurement of the higher-order Kerr indices at a wavelength of $800 \mathrm{~nm}$ up to $n_{8}$ in air and $n_{10}$ in argon [11] showed that they have alternate signs, and are therefore alternatively focusing and defocusing. Furthermore, by implementing these terms in a numerical simulation of filamentation, we have recently shown that the defocusing terms $n_{4}$ and $n_{8}$, rather than the plasma, provide the main regularizing process in the filamentation of 30 -fs pulses in air at $800 \mathrm{~nm}$ so that plasma generation and propagation equations are almost decoupled [12]. This finding provides an explanation for measurements of plasma-free filamentation $[13,14]$ and predicts symmetrical temporal pulse shapes, in contrast with a balance between the instantaneous Kerr term and the time-integrated plasma contribution, which implies strongly asymmetric pulse shapes [15]. It also explains

\footnotetext{
*jerome.kasparian@unige.ch
}

the discrepancy, by almost 2 orders of magnitude, between experimentally measured and numerically derived electron densities within filaments. While the experiments yield some $10^{14} \mathrm{~cm}^{-3}[16,17]$, the numerical simulations require a few $10^{16} \mathrm{~cm}^{-3}$ to balance the $n_{2} I$ Kerr self-focusing term $[3,4]$. The consideration of the higher-order Kerr terms also turned out to be necessary to obtain a quantitative agreement between numerical simulations and experimental results about the propagation of ultrashort infrared (IR) pulses in an argon-filled hollow-core fiber $[18,19]$.

However, the ionization rates are higher at shorter wavelengths so that ionization is generally believed to be much stronger in the case of ultraviolet (UV) filamentation [20], while its low efficiency in the IR would expectedly prevent self-guiding of filaments. But the recently derived generalized Miller formulas [21] predict larger absolute values of the higher-order nonlinear indices at shorter wavelengths, with faster spectral dependencies for the higher orders. As a consequence, the relative contribution to self-guiding cannot be easily extrapolated from qualitative discussions.

In this paper, we numerically investigate these relative contributions from the near UV to the near infrared (NIR). Using the values of the $n_{2 j}$ indices at any wavelength as obtained from the generalized Miller formulas [21], and the values recently measured at $800 \mathrm{~nm}$ [11], we simulate the filamentation of laser pulses from 300 to $1600 \mathrm{~nm}$ in air and argon. We show that filaments are efficiently generated at all wavelengths, even in the IR. Moreover, the plasma marginally contributes to self-guiding in the filamentation of 30-fs laser pulses in argon for wavelengths typically longer than $400 \mathrm{~nm}$. In air, where the ionization of oxygen is about 100 times more efficient than in argon, the Kerr terms still provide the largest defocusing contribution, although the plasma contribution can be neglected over the whole propagation length only in the IR. This finding strongly impacts the plasma generation. We also find that UV wavelengths are not optimal to maximize ionization in a filament.

Numerical simulations are performed, as described in detail in Ref. [12], by solving a nonlinear Schrödinger equation (NLSE) taking the higher-order Kerr terms into account. The reduced scalar envelope $\varepsilon$ defined such that $|\varepsilon(r, z, t)|^{2}=$ $I(r, z, t), I$ being the intensity, is assumed to vary slowly in 
time and along $z$ :

$$
\begin{aligned}
\partial_{z} \varepsilon= & \frac{i}{2 k} \Delta_{\perp} \varepsilon-i \frac{k^{\prime \prime}}{2} \partial_{t}^{2} \varepsilon \\
& +\left(1+\frac{i}{\omega} \frac{\partial}{\partial t}\right) i \frac{k}{n_{0}}\left(\sum_{j \geqslant 1} n_{2 j}|\varepsilon|^{2 j}\right) \varepsilon-i \frac{k}{2 n_{0}^{2} \rho_{c}} \rho \varepsilon \\
& -\frac{\varepsilon}{2} \sum_{\ell=\mathrm{O}_{2}, \mathrm{~N}_{2}}\left[\sigma_{\ell}(\omega) \rho+\frac{W_{\ell}\left(|\varepsilon|^{2}, \omega\right) U_{\ell}}{|\varepsilon|^{2}}\left(\rho_{\mathrm{nt}_{\ell}}-\rho\right)\right],
\end{aligned}
$$

where $\omega$ and $k=n_{0} \omega / c$ are the angular frequency and wave number of the carrier wave, $k^{\prime \prime}$ accounts for the linear groupvelocity dispersion (GVD), $\rho$ is the electron density, $\rho_{\mathrm{nt}_{\ell}}$ is the density of neutral molecules of species $\ell, \rho_{c}=m_{e} \epsilon_{0} \omega / q_{e}^{2}$ is the critical plasma density and $m_{e}$ and $q_{e}$ are the mass and charge of the electron, $n_{0}$ is the linear refractive index at $\lambda$, $W_{\ell}\left(|\varepsilon|^{2}\right)$ is the photoionization rate of species $\ell$ with ionization potential $U_{\ell}, \sigma_{\ell}$ is the cross section for avalanche ionization as defined below in Eq. (8), and $t$ refers to the retarded time in the reference frame of the pulse. The right-hand terms of Eq. (3) account for spatial diffraction, second-order GVD, Kerr self-focusing (including the self-steepening term), defocusing by the higher-order nonlinear refractive indices, plasma defocusing, inverse bremsstrahlung, and multiphoton absorption, respectively. We neglect the delayed orientational response, which for pulses longer than $100 \mathrm{fs}$ would increase the self-focusing term [3,4] and affect the ionization efficiency of $\mathrm{N}_{2}$ and $\mathrm{O}_{2}$ molecules by less than $20 \%$ and $10 \%$, respectively [22,23]. These opposite effects are negligible in the numerical simulations for $30-$ fs pulse duration, and do not affect qualitatively the following discussion even for longer pulses. We also neglect space-time focusing. However, in the following, we mainly focus on the self-guiding process, peak intensity, and ionization, which are little affected by this approximation.

The spectral dependence of the NLSE (3) stems from the plasma contribution as well as the dispersion of the linear $\left(n_{0}\right)$ and nonlinear $\left(n_{2 j}\right)$ refractive indices. The latter can be deduced at any frequency $\omega$ from those of $\mathrm{O}_{2}$ and $\mathrm{N}_{2}$ at the same frequency by following the Lorenz-Lorentz relation, which links the refractive index of a mix of nonpolar gases to its components [24],

$$
\frac{n_{\text {mix }}^{2}(\omega)-1}{n_{\text {mix }}^{2}(\omega)+2}=\sum \rho_{i} \frac{n_{i}^{2}(\omega)-1}{n_{i}^{2}(\omega)+2},
$$

where $n(\omega)$ is defined in Eq. (1) and $\rho_{i}$ denotes the relative abundance of the species $i$ in the mix. Considering that $\Delta n_{\text {Kerr }} \ll 1$, a first-order development of Eq. (4) yields, for any order $j$ :

$$
n_{2 j, \mathrm{Air}}(\omega)=0.79 n_{2 j, \mathrm{~N}_{2}}(\omega)+0.21 n_{2 j, \mathrm{O}_{2}}(\omega) .
$$

The values of the $n_{2 j}$ for argon, nitrogen, and oxygen can be calculated at any frequency $\omega$ from their values measured at $\lambda_{0}=800 \mathrm{~nm}$ [11] and the dispersion curves in those gases [25], through the generalized Miller formula [21] expressed in term of nonlinear refractive indices:

$$
n_{2 j}(\omega)=n_{2 j}\left(\omega_{0}\right)\left[\frac{n_{0}^{2}(\omega)-1}{n_{0}^{2}\left(\omega_{0}\right)-1}\right]^{2(j+1)} .
$$

While $n_{8 \text {,Air }}$ varies by a factor of 2 between 300 and $1600 \mathrm{~nm}$, ionization displays an even much steeper spectral dependence. More specifically, the temporal evolution of the electron density is given by

$$
\frac{\partial \rho}{\partial t} \approx \sum_{\ell=\mathrm{O}_{2}, \mathrm{~N}_{2}}\left[W_{\ell}(I, \omega) \rho_{0, \mathrm{nt}_{\ell}}+\frac{\sigma_{\ell}(\omega)}{U_{\ell}} I \rho\right],
$$

where $\rho_{0, \text { nt }_{\ell}}$ is the initial density of neutral molecules of species $\ell$. Here, attachment to neutral molecules and recombination with positive ions have been neglected owing to the short pulse durations considered in this paper. The cross section for avalanche ionization is calculated on the basis of Drude's theory [3]:

$$
\begin{aligned}
\sigma_{\ell}(\omega)= & \frac{q_{e}^{2}}{m_{e} \epsilon_{0} n_{0}(\omega) c v_{e, \ell}\left(1+\frac{\omega^{2}}{v_{e, \ell}^{2}}\right)} \\
& \approx \frac{q_{e}^{2} v_{e, \ell}}{\omega^{2} m_{e} \epsilon_{0} n_{0}(\omega) c}
\end{aligned}
$$

where $v_{e, \ell}$ is the mean-collisional frequency of an electron with the species $\ell$ (i.e., the average electron velocity, divided by the mean-free path of an electron, assuming that only species $\ell$ is present), and $c$ is the speed of light. At atmospheric pressure in air, $v_{e, \mathrm{O}_{2}}=1 /(1.75 \mathrm{ps}), v_{e, \mathrm{~N}_{2}}=1 /(440 \mathrm{fs})$, and $v_{e, \mathrm{Ar}}=$ $1 /(350 \mathrm{fs})$. The multiphoton and tunnel ionization rates are given by the multispecies generalized Keldysh-Perelomov, Popov, Terent'ev formulation $[3,16]$,

$$
\begin{aligned}
W_{\ell}\left(|\varepsilon|^{2}, \omega\right)= & \frac{4 \sqrt{2}}{\pi}\left|C_{n_{\ell}^{*}, l_{\ell}^{*}}\right|^{2}\left(\frac{4 \sqrt{2} U_{\ell}^{3 / 2}}{E_{p} \sqrt{1+\gamma_{\ell}^{2}}}\right)^{2 n_{\ell}^{*}-3 / 2-\left|m_{\ell}\right|} \\
& \left.\times \frac{f\left(l_{\ell}, m_{\ell}\right)}{\left|m_{\ell}\right| !} e^{-2 v_{\ell}\left(\sinh ^{-1}\left(\gamma_{\ell}\right)-\frac{\gamma_{\ell} \sqrt{\gamma_{\ell}^{2}+1}}{1+2 \gamma_{\ell}^{2}}\right.}\right) \\
& \times U_{\ell} \frac{\gamma_{\ell}^{2}}{1+\gamma_{\ell}^{2}} \sum_{\kappa_{\ell} \geqslant v_{\ell}}^{+\infty} e^{-\alpha_{\ell}\left(\kappa_{\ell}-v_{\ell}\right)} \Phi_{m}\left(\sqrt{\beta_{\ell}\left(\kappa_{\ell}-v_{\ell}\right)}\right),
\end{aligned}
$$

where, expressed in atomic units, $E_{p}=|\varepsilon| \sqrt{2 /\left(\epsilon_{0} c\right)}, \gamma_{\ell}=$ $\omega \sqrt{2 U_{\ell}} / E_{p}, v_{\ell}=U_{\ell}\left[1+1 /\left(2 \gamma^{2}\right)\right] /(\hbar \omega), \beta_{\ell}=2 \gamma_{\ell} / \sqrt{1+\gamma_{\ell}^{2}}$, $\alpha_{\ell}=2\left[\sinh ^{-1}\left(\gamma_{\ell}\right)-\gamma_{\ell} / \sqrt{1+\gamma_{\ell}^{2}}\right], \quad$ and $\Phi_{m}(x)=e^{-x^{2}} \int_{0}^{x}$ $\left(x^{2}-y^{2}\right)^{\left|m_{\ell}\right|} e^{y^{2}} d y . n_{\ell}^{*}=Z_{\ell} / \sqrt{2 U_{\ell}}$ is the effective quantum number, $l_{\ell}^{*}=n_{\ell}^{*}-1, l_{\ell}$, and $m_{\ell}$ are the orbital momentum and the magnetic quantum number, respectively. In air, $l_{\ell}=m_{\ell}=0$ [26]. $Z_{\ell}$ is the residual ion charge accounting for the difference between the $\mathrm{O}_{2}$ and $\mathrm{N}_{2}$ molecules and their atomic counterparts. These empirical coefficients $Z_{\mathrm{O}_{2}}=0.53$ and $Z_{\mathrm{N}_{2}}=0.9$ have been measured at $800 \mathrm{~nm}$ [27] and are expected to be independent from the wavelength. Since argon 
is an atomic gas, $Z_{\mathrm{Ar}}=1$. The factors $\left|C_{n_{\ell}^{*}, l_{\ell}^{*}}\right|$ and $f\left(l_{\ell}, m_{\ell}\right)$ are

$$
\begin{gathered}
\left|C_{n_{\ell}^{*}, l_{\ell}^{*}}\right|=\frac{2^{2 n_{\ell}^{*}}}{n_{\ell}^{*} \Gamma\left(n_{\ell}^{*}+l_{\ell}^{*}+1\right) \Gamma\left(n_{\ell}^{*}-l_{\ell}^{*}\right)}, \\
f\left(l_{\ell}, m_{\ell}\right)=\frac{\left(2 l_{\ell}+1\right)\left(l_{\ell}+\left|m_{\ell}\right|\right) !}{2^{\left|m_{\ell}\right|}\left|m_{\ell}\right| !\left(l_{\ell}-\left|m_{\ell}\right|\right) !} .
\end{gathered}
$$

To quantify the relative contributions of the higher-order Kerr terms $\left(n_{4}-n_{10}\right)$ and of the ionization to the defocusing, which balances the self-focusing, we define the instantaneous ratio $\xi$ between the refractive index changes induced by both of these contributions at any location $\vec{r}$ :

$$
\xi(\vec{r}, t)=\left|\sum_{j \geqslant 2} n_{2 j} I(\vec{r}, t)^{j}\right| / \frac{\rho(\vec{r}, t)}{2 n_{0} \rho_{c}} .
$$

This expression is, in fact, the ratio of the magnitude of the two defocusing terms in the propagation equation (3), namely, those accounting for higher-order Kerr terms and plasma defocusing, respectively. When considering the overall action of both effects on the whole pulse duration, we define a pulse-integrated value of $\xi$ :

$$
\bar{\xi}(\vec{r})=\int\left|\sum_{j \geqslant 2} n_{2 j} I(\vec{r}, t)^{j}\right||\varepsilon(\vec{r}, t)| d t / \int \frac{\rho(\vec{r}, t)}{2 n_{0} \rho_{c}}|\varepsilon(\vec{r}, t)| d t .
$$

We numerically integrated the propagation equation (3) for an ultrashort pulse typical of laboratory-scale filamentation experiments: 30-fs Fourier-limited full width at half-maximum pulse duration, a peak power of 6.5 critical powers $P_{\mathrm{cr}}=$ $\lambda^{2} / 4 \pi n_{2}$, a beam diameter of $\sigma_{r}=4 \mathrm{~mm}$, a focal length $f=1 \mathrm{~m}$, and a pressure of 1 bar of either air or argon. The main results are displayed in Fig. 1.

Our simulations yield filamentation over the whole investigated spectral range $(300-1600 \mathrm{~nm})$, reproducing experimental observations from the UV [28,29] to the mid IR [30]. As visible in Figs. 1(a) and 1(b), the clamping intensity, filament onset, and filament length are very similar in air and argon over the whole considered spectral length for a given incident reduced power $P / P_{\mathrm{cr}}$. Moreover, longer wavelengths yield longer filaments with an earlier onset. Furthermore, the output spectrum after $1.5-\mathrm{m}$ propagation [panels (e) and (f)] is broader for longer wavelengths. As a result, the relative broadening, defined as the ratio of the output spectral width to the initial frequency $\Delta \omega / \omega_{0}$ is almost constant across the spectrum. Spectral properties appear very similar in air and argon.

In contrast, the electron density is approximately ten times higher in the former than in the latter. The spectral dependence of the electron density is very nonmonotonic [panels (c) and (d)]. On one hand, due to steps in the number of photons required for ionization, the electron density is not a monotonic function of the wavelength. For instance, ionization in air is almost two times more efficient at $793 \mathrm{~nm}$ than at $815 \mathrm{~nm}$ (see Fig. 2). On the other hand, contrary to expectations that shorter wavelengths should result in stronger ionization, a maximum in the peak electron density is observed in the 610-nm range in the case of air, and around $470 \mathrm{~nm}$ in argon [Figs. 1(c), 1(d), and 4(a)]. Away from this maximum, the peak electron density

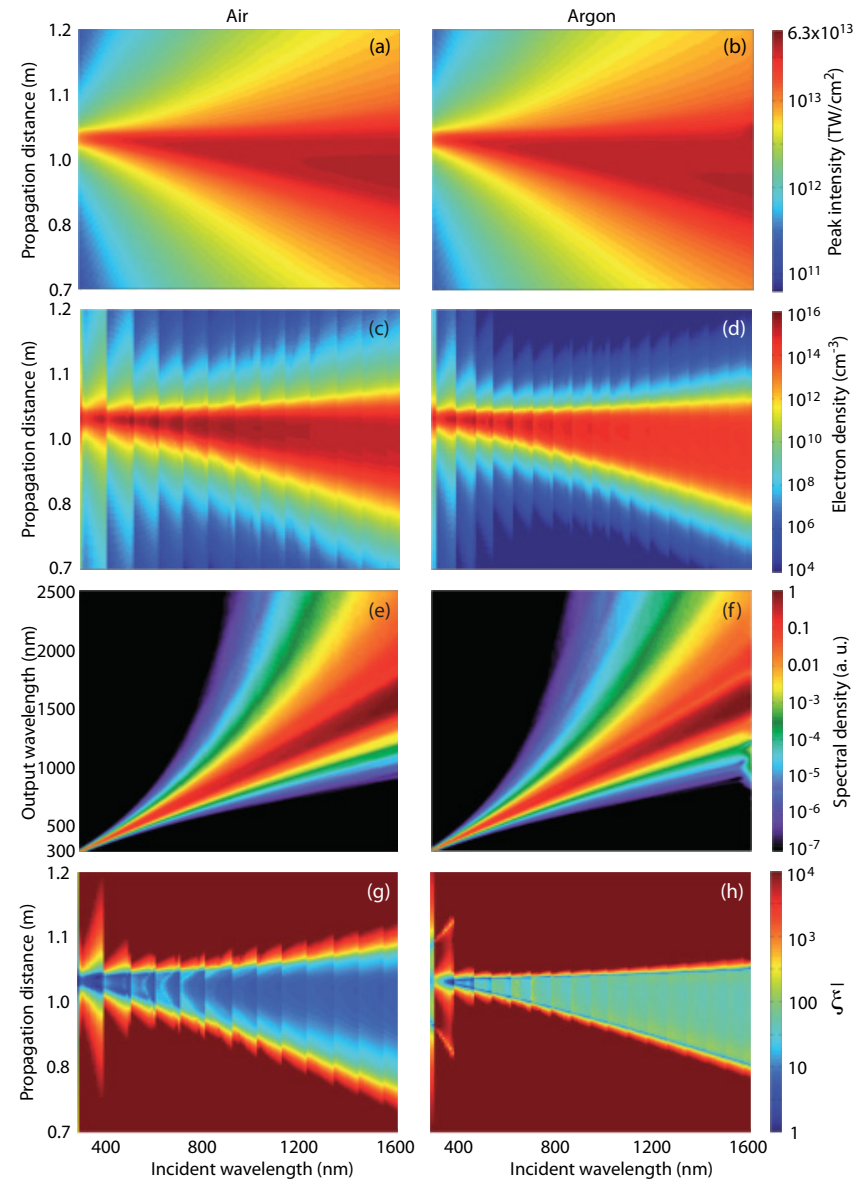

FIG. 1. (Color) Maximum on-axis intensity (a), (b), electron density (c), (d), spectral broadening (e), (f), and pulse-averaged ratio $\bar{\xi}$ of the on-axis pulse-averaged contributions of the higher-order Kerr and plasma contributions to the nonlinear refractive index [(g), (h), see text for details], as a function of the wavelength and propagation distance in air (a), (c), (e), (g) and argon (b), (d), (f), (h).

decreases within a dynamics of typically 1 order of magnitude. This unexpected behavior stems from the convolution of the respective spectral dependences of (i) the ionization rate $W$, which increases at shorter wavelengths and (ii) the peak intensity, which decreases in the UV. This decrease is due to the fact that, according to Eq. (6) and considering the typical dispersion curves in gases, the higher-order indices increase faster, in absolute values, when the frequency increases. As a consequence, the clamping intensity canceling the Kerr effect $I_{\Delta n_{\text {Kerr }}=0}$ is lower, as shown in Fig. 3.

The spectral dependence of the peak electron density by more than 1 order of magnitude, also evidenced in Fig. 4(a), appears in contradiction with numerical results obtained without considering the higher-order Kerr terms [31], which predict almost similar electron densities, close to $10^{16} \mathrm{~cm}^{-3}$, at the three investigated wavelengths $(248,800$, and $1550 \mathrm{~nm})$. Since the latter results were obtained with longer pulses (127 fs) of slightly lower power than in our paper, a direct quantitative comparison cannot be performed. However, we expect that the qualitative difference between our results and those of Ref. [31] are due to the inclusion of the higher-order 


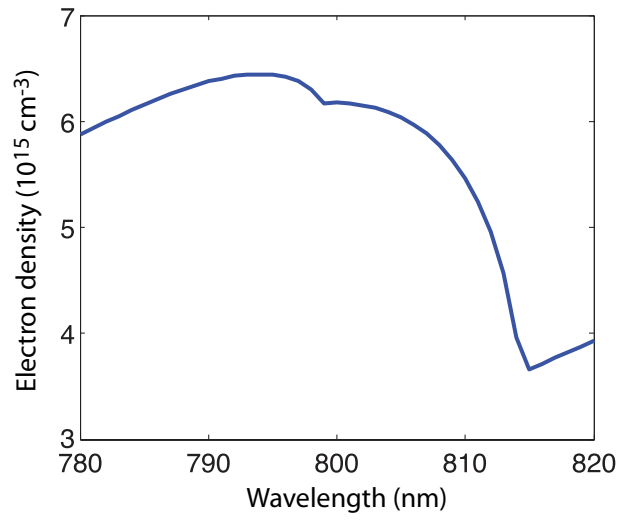

FIG. 2. (Color online) Wavelength dependence close to $800 \mathrm{~nm}$ of the electron density generated by a 30 -fs pulse of constant intensity $50 \mathrm{TW}$.

Kerr terms in our model, and thus illustrate the influence of the latter.

The very similar behavior, except for the plasma density, of two gases with comparable values of the nonlinear refractive indices but significantly different ionization potentials, suggests that filamentation is driven by the higher-order Kerr terms rather than by plasma, not only at $800 \mathrm{~nm}$ as evidenced recently [12], but also on the whole investigated spectral range in both air and argon. Indeed, the on-axis $\bar{\xi}$ ratio [Figs. 1(g), and 1(h)] exceeds 1 in all considered conditions. Furthermore, the higher-order Kerr terms strongly dominate the filamentation dynamics $(\bar{\xi}>10)$ at all wavelengths above $\lambda \sim 400 \mathrm{~nm}$ in argon and above $\lambda \sim 600 \mathrm{~nm}$ in air, although, due to the thresholds induced by the steps in photon numbers required to ionize oxygen [see Eq. (10)], the evolution of this behavior is not strictly monotonic. Due to the domination of the higher-order Kerr terms, the latter will govern the intensity within the filaments so that the ionization is mostly decoupled from the filamentation dynamics.

It should be noted that Miller formulas are sometimes considered to underestimate dispersion [32]. However, such correction to the dispersion curve of higher-order Kerr terms would result in a larger absolute value of the high-order Kerr

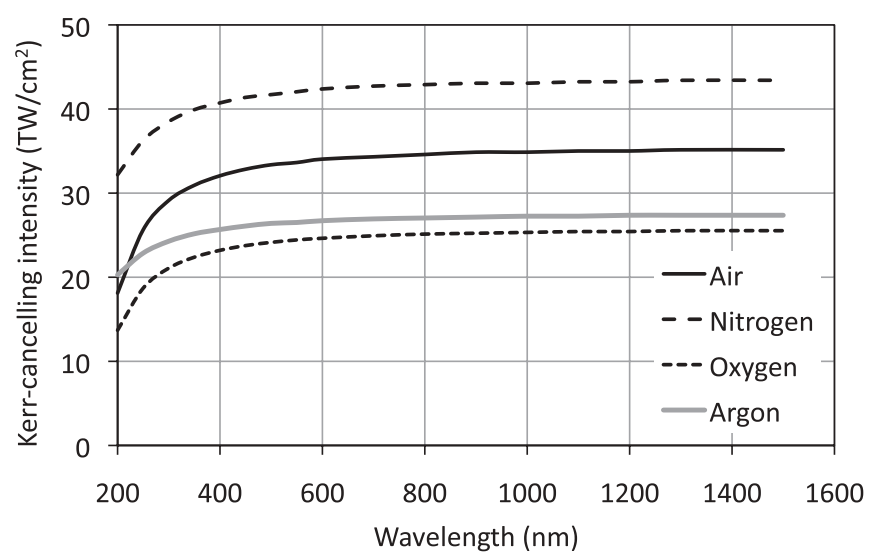

FIG. 3. Wavelength dependence of the clamping intensity canceling the Kerr effect $I_{\Delta n_{\text {Kerr }}=0}$, in $\mathrm{O}_{2}, \mathrm{~N}_{2}$, air, and Ar, based on the dispersion of the higher-order Kerr indices from Eq. (6).
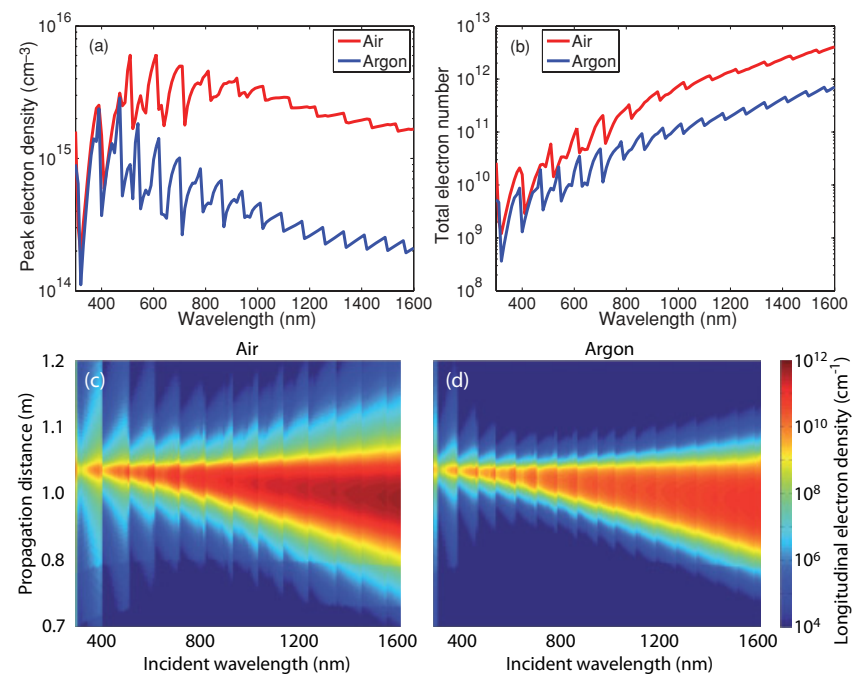

FIG. 4. (Color) Wavelength dependence of ionization in laser filamentation. (a) Peak electron density; (b) total number of electrons; (c), (d) transverse-integrated longitudinal electron density in air and argon, representative of the electric conductivity of the filament. Note the difference with Figs. 1(c) and 1(d), which is due to the larger filament diameter in the IR.

indices in the UV. In this case, $I_{\Delta n_{\text {Kerr }}=0}$ will be even lower in the UV. In other words, the curves of Fig. 3 will decrease faster on the left side of the graph. As a consequence, the higher-order Kerr indices will have an even more dominant contribution to the self-guiding, as compared to that of plasma. Such larger dispersion would therefore qualitatively reinforce our conclusion about the domination of defocusing higher-order Kerr terms over the plasma defocusing.

This new vision of filamentation provides straightforward interpretation for experimental observations that the spectral broadening mostly occurs at the beginning of the filaments, while the spectrum is little affected in the main region of self-guided propagation [33]. This is due to the fact that, in the self-guided propagation, the intensity is clamped close to $I_{\Delta n_{\text {Kerr }}=0}$ so that the spectral counterpart of this Kerr effect, self-phase modulation, is also waved out. The earlier onset of filamentation for longer wavelengths is compatible with the Marburger formula [34], which predicts that the nonlinear focal length describing self-focusing is inversely proportional to the wavelength. Furthermore, the longer filaments in the IR appear to stem from the lower peak electron density [Fig. 4(a)].

The very smooth dispersion curve of both air and argon above $500 \mathrm{~nm}$ [21] results in a very slow variation of this clamping intensity $I_{\Delta n_{\text {Kerr }}=0}$ (Fig. 3) and explains the quasiconstant clamping intensity observed over this spectral range.

The availability of simulations at constant reduced power $P / P_{\mathrm{cr}}$ over a broad spectral range allows to discuss the choice of wavelength to optimize the filaments properties for specific purposes. As described earlier, the spectral dependence of the electron generation contrasts strongly with the common expectation that, due to a more efficient ionization, UV wavelengths should ionize the propagation medium more efficiently. 
However, the peak electron density is not directly relevant for typical atmospheric applications. For example, laserassisted water condensation [35], can be expected to require the largest possible value of the total generated charge. This total charge is obtained by integrating the plasma density over the filament length and cross section [Fig. 4(b)]. Here, the longer filaments in the IR as well as their larger diameters result in an unanticipated larger total charge at longer wavelengths.

Applications such as lightning control [36] require the longest possible filaments with the higher conductivity [i.e., a high longitudinal (transversely integrated) electron density]. Long wavelengths simultaneously optimize both of these properties [Figs. 4(c) and 4(d)]. In that regard, UV lasers providing short filaments with a small diameter and decreasing transverse-integrated electron densities would expectedly be less efficient than the commonly used titanium:sapphire lasers around $800 \mathrm{~nm}$, while wavelengths further in the IR should even be more efficient. IR is also very attractive because it would allow to work in the telecommunication spectral region $(1.55 \mu \mathrm{m})$, where optical components are available and eyesafety standards are much more favorable for atmospheric applications than in the rest of the NIR and visible spectral region.

In conclusion, applying generalized Miller formulas to estimate arbitrary-order nonlinearities from the IR to the UV spectrum, we have shown that higher-order nonlinear indices (up to $n_{8}$ in air and $n_{10}$ in argon) have a dominant contribution to both the focusing and the defocusing terms implied in the self-guiding of 30-fs laser pulses in air, at all wavelengths between 300 and $1600 \mathrm{~nm}$. As a consequence, the plasma generation is almost decoupled from the self-guiding of filaments over the whole visible and IR spectral range. Instead, filaments can be considered as a self-guiding regime mostly governed in air and argon by the balance between the alternate signs of the nonlinear indices, respectively resulting in Kerr self-focusing and self-defocusing.

Moreover, a systematic investigation at constant reduced power $P / P_{\text {cr }}$ as a function of wavelength provides hints to choose optimal wavelengths for generating filaments optimized for a specific application. In particular, we have shown that, contrary to previous expectations, the IR is more favorable than the commonly used 800-nm wavelength if long ionized filaments or high total amounts of charges are required. This raises the hope to further improve the spectacular results $[5,35,36]$ recently obtained with titanium:sapphire lasers. Moreover, the higher eye-safety threshold in the telecommunication window at $1.55 \mu \mathrm{m}$ is favorable for the practical development of the envisioned applications in free space.

This work was supported by the Conseil Régional de Bourgogne, the ANR COMOC, the FASTQUAST ITN Program of the 7th FP, and the Swiss NSF (Contracts No. 200021116198 and No. 200021-125315).
[1] A. Braun, G. Korn, X. Liu, D. Du, J. Squier, and G. Mourou, Opt. Lett. 20, 73 (1995).

[2] S. L. Chin, S. A. Hosseini, W. Liu, Q. Luo, F. Theberge, N. Aközbek, A. Becker, V. P. Kandidov, O. G. Kosareva, and H. Schroeder, Can. J. Phys. 83, 863 (2005).

[3] L. Bergé, S. Skupin, R. Nuter, J. Kasparian, and J.-P. Wolf, Rep. Prog. Phys. 70, 1633 (2007).

[4] A. Couairon and A. Mysyrowicz, Phys. Rep. 441, 47 (2007).

[5] J. Kasparian and J.-P. Wolf, Opt. Express 16, 466 (2008).

[6] N. Aközbek, M. Scalora, C. M. Bowden, and S. L. Chin, Opt. Commun. 191, 353 (2001).

[7] A. Vinçotte and L. Bergé, Phys. Rev. A 70, 061802(R) (2004).

[8] G. Fibich and B. Ilan, Opt. Lett. 29, 887 (2004).

[9] L. Bergé, S. Skupin, G. Méjean, J. Kasparian, J. Yu, S. Frey, E. Salmon, and J. P. Wolf, Phys. Rev. E 71, 016602 (2005).

[10] J.-F. Zhang, Q. Tian, Y.-Y. Wang, C.-Q. Dai, and L. Wu, Phys. Rev. A 81, 023832 (2010).

[11] V. Loriot, E. Hertz, O. Faucher, and B. Lavorel, Opt. Express 17, 13429 (2009); 18, 3011(E) (2010).

[12] P. Béjot, J. Kasparian, S. Henin, V. Loriot, T. Vieillard, E. Hertz, O. Faucher, B. Lavorel, and J.-P. Wolf, Phys. Rev. Lett. 104, 103903 (2010).

[13] G. Méchain et al., Appl. Phys. B 79, 379 (2004).

[14] A. Dubietis, E. Gaizauskas, G. Tamosauskas, and P. Di Trapani, Phys. Rev. Lett. 92, 253903 (2004).

[15] G. Stibenz, N. Zhavoronkov, and G. Steinmeyer, Opt. Lett. 31, 274 (2006).

[16] J. Kasparian, R. Sauerbrey, and S. L. Chin, Appl. Phys. B: Lasers Opt. 71, 877 (2000).

[17] F. Théberge, W. Liu, P. T. Simard, A. Becker, and S. L. Chin, Phys. Rev. E 74, 036406 (2006).
[18] B. E. Schmidt et al., Appl. Phys. Lett. 96, 121109 (2010).

[19] P. Béjot, B. E. Schmidt, J. Kasparian, J.-P. Wolf, and F. Legaré, Phys. Rev. A 81, 063828 (2010).

[20] J. Schwarz, P. Rambo, and J.-C. Diels, Opt. Commun. 180, 383 (2000).

[21] W. Ettoumi, Y. Petit, J. Kasparian, and J.-P. Wolf, Opt. Express 18, 6613 (2010).

[22] I. V. Litvinyuk, K. F. Lee, P. W. Dooley, D. M. Rayner, D. M. Villeneuve, and P. B. Corkum, Phys. Rev. Lett. 90, 233003 (2003).

[23] Z. X. Zhao, X. M. Tong, and C. D. Lin, Phys. Rev. A 67, 043404 (2003).

[24] C. J. F. Böttcher, Theory of Electric Polarisation (Elsevier, Amsterdam, 1978).

[25] J. Zhang, Z. H. Lu, and L. J. Wang, Appl. Opt. 47, 3143 (2008).

[26] R. Nuter and L. Bergé, J. Opt. Soc. Am. B 23, 874 (2006).

[27] A. Talebpour, J. Yang, and S. L. Chin, Opt. Commun. 163, 29 (1999).

[28] J. Schwarz, P. Rambo, J.-C. Diels, M. Kolesik, E. M. Wright, and J. V. Moloney, Opt. Commun. 180, 383 (2000).

[29] S. Tzortzakis, B. Lamouroux, A. Chiron, M. Franco, B. Prade, A. Mysyrowicz, and S. D. Moustaizis, Opt. Lett. 25, 1270 (2000).

[30] C. P. Hauri et al., Opt. Lett. 32, 868 (2007).

[31] S. Skupin and L. Bergé, Opt. Commun. 280, 173 (2007).

[32] V. Mizrahi and D. P. Shelton, Phys. Rev. Lett. 55, 696 (1985).

[33] D. Mondelain, Ph.D. thesis, Université Lyon I, 2001 (unpublished) [http://tel.archives-ouvertes.fr/tel-00396346/fr/].

[34] E. L. Dawes and J. H. Marburger, Phys. Rev. 179, 862 (1969).

[35] P. Rohwetter et al., Nature Photon. 4, 451 (2010).

[36] J. Kasparian et al., Opt. Express 16, 5757 (2008). 